\title{
Неоднородные процессы инжекции и теплопереноса в реверсивно-включаемых динисторах при работе в частотно-импульсных режимах с ограниченным теплоотводом
}

\author{
(C) А.В. Горбатюк ${ }^{1}$, Б.В. Иванов ${ }^{2}$ \\ ${ }^{1}$ Физико-технический институт им. А.Ф. Иоффе Российской академии наук, \\ 194021 Санкт-Петербург, Россия \\ ${ }^{2}$ Санкт-Петербургский государственный электротехнический университет „ЛЭТИ“, \\ 197376 Санкт-Петербург, Россия \\ E-mail: agor.pulse@mail.ioffe.ru
}

(Получена 22 октября 2018 г.

Принята к печати 29 октября 2018 г.

Принята к публикации 29 октября 2018 г.)

Впервые получено самосогласованное расчетно-теоретическое описание физических процессов в реверсивно-включаемых динисторах (РВД) при работе в частотно-импульсных режимах с ограниченным теплоотводом. Обосновано упрощенное представление нелинейного многомасштабного механизма взаимодействия между инжекционной и теплоотводящей подсистемами, и на этой основе разработан метод расчета максимальной частоты РВД-коммутатора. Рассчитана зависимость допустимой частоты от мощности охладителя для заданных параметров чипа и формы коммутируемых импульсов. Показано, что при обеспечении надлежащего теплоотвода единичные РВД-модули с площадью чипа $1 \mathrm{~cm}^{2}$ и рабочим напряжением $U \approx 2.5$ кВ будут способны переключать энергию 0.25 Дж за импульс с частотой повторения до 30 кГц. Для высоковольтных генераторов на их основе с $U \approx 100$ кВ передаваемая в нагрузку мощность на этой частоте составит несколько долей МВт.

DOI: 10.21883/FTP.2019.04.47454.9009

\section{1. Введение}

Реверсивно-включаемые динисторы (РВД) [1-3], известные как особо мощные и надежные моноимпульсные переключатели микросекундного диапазона [4-7], в последние годы начинают применяться в высоковольтных частотных генераторах субмикросекундных импульсов $[8,9]$. Перспективной основой для этого могут послужить новые модификации РВД с оптимизированной структурой и экономичным способом реверсивной накачки $[10,11]$, численное моделирование которых обнаруживает более высокие показатели по сравнению с описанными в $[8,9]$ прототипами. Так, реализация предлагаемых вариантов в частотных цепях с рабочим напряжением $U \approx 2.5-5.0$ кВ при надлежащем охлаждении могла бы обеспечить коммутацию импульсов тока амплитудой $\sim(1-2)$ кА и длительностью в сотни наносекунд при непрерывной их генерации в нижнем килогерцовом диапазоне. Однако для высоковольтных генераторов $(\geq 100 \kappa \mathrm{B})$, как показали наши недавние оценки в [12], вопрос о предельно допустимых частотах оказывается весьма нетривиальным из-за проблем распределенного охлаждения конструкций и должен обсуждаться с учетом их конкретной специфики.

Действительно, коммутирующие узлы высоковольтных генераторов (высоковольтные сборки) составляются из большого числа отдельных переключающих полупроводниковых чипов, перемежающихся с теплопроводящими пластинами для поперечного выноса тепла из объема во внешний радиатор (см. пример на рис. 8 работы [13]).
Поэтому в каждом чипе должно наблюдаться определенное снижение температуры от центра к периферии, что из-за сильных температурных зависимостей инжекции может отразиться на степени модуляции проводимости, на плотности тока и энергии джоулевых потерь в объеме чипа и в итоге существенно повлиять на диапазон допустимых частот генерации.

Применительно к частотным модификациям РВД строгий количественный анализ пространственно неоднородных электротепловых эффектов, необходимый для создания научного базиса для практического освоения этого класса приборов, ранее не проводился, и мы ставим его выполнение целью настоящего исследования.

\section{2. Многоуровневая формулировка пространственно-неоднородной электротепловой задачи}

Будем считать, что все последовательные звенья высоковольтной сборки - единичные коммутирующие РВД-модули - находятся в одинаковых физических условиях. Типичная геометрия модуля имеет вид цилиндра радиусом $r_{m}$, который состоит из находящихся в хорошем электротепловом контакте пластин чипа РВД $(\mathbf{S})$ и металлического теплоотвода $(\mathbf{T})$ толщиной $w$ и $h$ каждая (рис. $1, a)$.

Слой $\mathbf{S}$ является основным функциональным элементом модуля, и для его адекватного описания требуется учет того же набора законов электростатики и нели- 

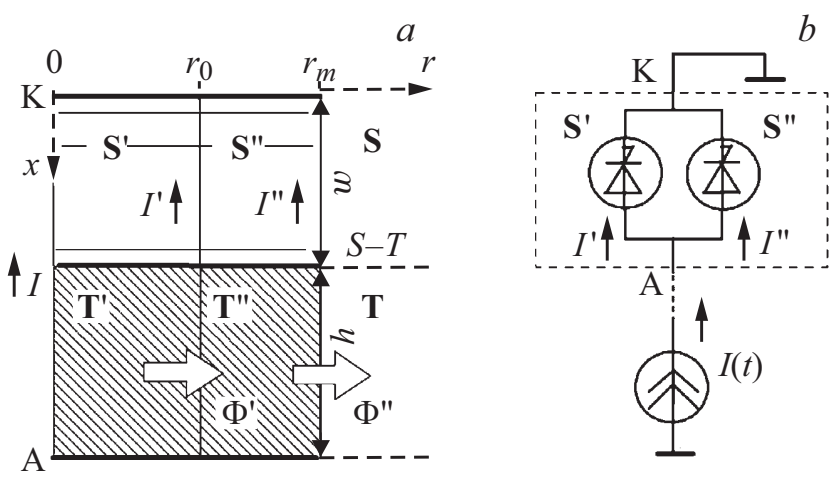

Рис. 1. Объемное представление электротепловой блок-схемы единичного модуля высоковольтной сборки РВД $(a)$ и эквивалентная электрическая цепь для изохронного компьютерного моделирования инжекционных процессов в разнородных сегментах РВД $(b)$.

нейного электронно-дырочного переноса с рекомбинацией и генерацией носителей, что был использован при имитационном моделировании субмикросекундных РВД в работах $[10,11]$. Однако механизм функционирования РВД-модуля в целом теперь представляется гораздо более сложным относительно случаев в $[10,11]$ из-за появления новых, трудно сопоставимых пространственных и временны́х масштабов. Радиус рабочей области модуля сильно превосходит по размеру все другие параметры реальной геометрии, включая размеры дискретных элементов ячеистой интегральной структуры РВД, $r_{m} \gg \Sigma w_{k}+h>d_{0}$, где $w_{k}$ - толщины отдельных слоев вертикального $P N P N$-профиля легирования и $d_{0}-$ расстояние между шунтами эмиттеров $[10,11]$. Поэтому перепады температуры по радиусу должны многократно превышать перепады по вертикальному измерению $\delta T(r) \gg \delta T(x) \sim 0$. Что касается временны́х масштабов, то они предопределены как физикой РВД, так и техническими особенностями частотно-импульсного режима. Времена многостадийного развития инжекции и тепловыделения в $\mathbf{S}$-слое за один импульс составляют $10^{-9}-10^{-6}$ с. Время вертикального перераспределения тепла по $x$ между слоями $\mathbf{S}$ и $\mathbf{T}$ задается периодом цикла $\tau_{f}=f^{-1} \sim\left(10^{-4}-10^{-3}\right)$ с, где $f$ - частота повторения импульсов. Распределенный по $r$ съем тепла, циклически пополняемого в Т-слое, и его поперечный вывод в радиатор, сопровождающийся временны́м усреднением и установлением квазистатического профиля $T_{\text {stat }}(r)$, затягивается до $10^{-1} \mathrm{c}$.

Получить полное самосогласованное описание столь сложной пространственно-неоднородной и многомасштабной электротепловой задачи за рациональный период времени не представляется возможным из-за необходимости использования чрезвычайно густой дискретизации координатно-временны́х расчетных сеток. Поэтому мы будем придерживаться классической логики количественного исследования больших нелинейных систем [14] и начнем с построения упрощенного, но структурно-замкнутого решения, выделяя в общей формулировке ограниченное число доминирующих по влиянию функциональных частей объема, нелокально взаимодействующих между собой через смежные границы.

Следуя этому подходу, разделим геометрию единичного звена по радиусу на коаксиальные сегменты $0<r<r^{\prime}$ и $r^{\prime}<r<r^{\prime \prime}=r_{m}$, соответствующие центральной и периферийной частям чипа $\left(\mathbf{S}^{\prime}\right.$ и $\left.\mathbf{S}^{\prime \prime}\right)$ и примыкающим к ним участкам теплоотвода $\left(\mathbf{T}^{\prime}\right.$ и $\left.\mathbf{T}^{\prime \prime}\right)$. Будем считать, что в связи с заведомо высокой листовой теплопроводностью слоя $\mathbf{T}$ радиальный вывод тепла за пределы РВД-модуля осуществляется только за счет радиальных потоков $\Phi$ по этому слою (см. рис. 1). Для установившегося частотного режима интегральная величина потока $\Phi^{\prime \prime}$ через цилиндрическую поверхность внешней границы $\mathbf{T}^{\prime \prime}$, задающая требуемую для охлаждения одного звена мощность внешнего охладителя, должна равняться усредненной по времени полной мощности импульсной генерации тепла в слое $\mathbf{S}$.

Далее реальное распределение $T(t, r)$, связанное с конкретной величиной $\Phi^{\prime \prime}$, будет аппроксимировано прямоугольной ступенькой с усредненными по периоду $\tau_{f}$ и по объемам сегментов $\mathbf{S}^{\prime}$ и $\mathbf{S}^{\prime \prime}$ значениями температуры с перепадом $\delta T=T^{\prime}-T^{\prime \prime}>0$. Процедура согласования задаваемых величин $\delta T\left(T^{\prime}, T^{\prime \prime}\right)$ и интенсивности охлаждения будет описана позже при расчете допустимых частот генерации.

\section{3. Моделирование динамики зарядов и теплопереноса в РВД-модуле при радиальном перепаде температуры}

\section{1. Нелокальная аппроксимация механизма радиального перераспределения тока}

Численное описание перераспределения инжекционных токов и тепловыделения по площади РВД-чипа осуществлялось в приближении изохронного взаимодействия параллельно-связанных коаксиальных сегментов по схеме рис. $1, b$, предполагающей выполнение закона Кирхгоффа для полного тока модуля и токов сегментов: $I=I^{\prime}+I^{\prime \prime}$. Ввиду плотного размещения большого числа ( 10 4 единиц) маленьких шунтированных ячеек в типовых РВД (см. $[10,11])$, а также сильного ослабления влияния точечных шунтов при больших токах в расчетах предполагалось, что оба сегмента $\mathbf{S}$-слоя „технологически“ однородны по радиусу, а инжекционные процессы в каждом из них одномерны по вертикальному направлению $x$.

Для адекватного описания динамики распределенных по $x$ потенциалов, электрического поля, концентраций электронов и дырок и создаваемых ими потоков зарядов в сегментах $\mathbf{S}^{\prime}$ и $\mathbf{S}^{\prime \prime}$, подчиняющихся самосогласованной системе уравнения Пуассона и нестационарных уравнений непрерывности токов носителей, был ис- 
пользован имитационный программный продукт TCAD SENTAURUS [15].

В качестве опорной для сравнения была выбрана шестислойная $N^{+} P^{\prime} P N_{0} N^{\prime} P^{+}$-структура РВД, совпадающая по конфигурации вертикального (от катода к аноду) профиля легирования $N_{D}(x)$ с вариантом РВД-1 из работы [10]. Варьируемые значения поверхностной концентрации $N_{s}$ профиля легирования глубокой части $P$-базы катодного транзистора далее конкретизируются в тексте.

Для удобства и простоты интерпретации эффективная рабочая площадь всего чипа во всех случаях при выполнении расчетов принималась равной $A=1 \mathrm{~cm}^{2}$. Площади сегментов были заданы одинаковыми и равными $A^{\prime}=A^{\prime \prime}=A / 2$.

Форма импульса тока через контакты А и К задавалась близкой к исследуемой в [10]. Для стадии $-t_{R}<t<0$, где $t_{R}$ - время накачки, она трапецеидальна (рис. 2). Ее параметры уточнялись в вычислениях для примеров с различными пробными значениями заряда реверсивной накачки

$$
Q_{R}=\int|I(t)| d t
$$

Для стадии коммутации $t>0$ использовалась формула

$$
I(t)=I_{0} \exp \left(-t / t_{\text {rel }}\right) \sin \left(2 t / t_{\mathrm{F}}\right)
$$

с коэффициентами $I_{0}=2.47 \mathrm{\kappa A}, \quad t_{\text {rel }}=200 \mathrm{Hc} \quad$ и $t_{\mathrm{F}}=310$ нс. Такая форма тока практически совпадает с рассчитанной для РВД-1 в [10] в цепи с емкостью $C=78.3 \mathrm{H} \Phi / \mathrm{cm}^{2}, \quad$ заряженной до напряжения $U_{0}=2.5 \mathrm{\kappa B}$, с сопротивлением нагрузки $R=1 \mathrm{OM} \cdot \mathrm{cm}^{2}$ и остаточной индуктивностью $L=100 \mathrm{H}$ Н $\cdot \mathrm{cm}^{2}$ (начальная энергии конденсатора при этом равна $W_{C 0}=245$ мДж/ $\left.\mathrm{cm}^{2}\right)$.

Переходное анодное напряжение $U(t)$, одинаковое для обоих сегментов, являясь искомой переменной моделирования, затем используется в вычислении интегралов джоулевых потерь и их разделенных по сегментам частей по формуле

$$
W(t)=\int I U d t .
$$

На рис. 2 представлены результаты имитационных расчетов динамических переменных задачи для случая с профилем легирования катодного транзистора, показанным на рис. 3, $a$. Сплошными кривыми $1-3$ на рис. 2 обозначены соответственно временны́е зависимости падения напряжения $U(t)$ между контактами А и $\mathrm{K}$, полного тока $I(t)$ и текущего значения интеграла джоулевых потерь - см. (3). Текущие значения частей этих переменных $I^{\prime}, W^{\prime}$ и $I^{\prime \prime}, W^{\prime \prime}$ в центральном, $\mathbf{S}^{\prime}$, и периферийном, $\mathbf{S}^{\prime \prime}$, сегментах представлены кривыми $2^{\prime}, 3^{\prime}$ и $2^{\prime \prime}, 3^{\prime \prime}$.

Как и ожидалось, наблюдается опережающий рост текущих значений $I^{\prime \prime}(t)$ над $I^{\prime}(t)$. Различие между ними,

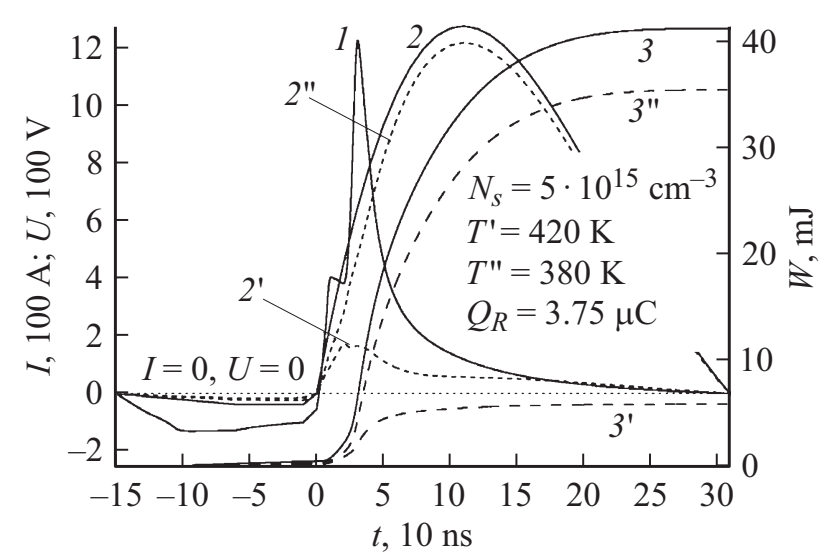

Рис. 2. Переходные характеристики чипа РВД и его составных частей (пояснения в тексте).
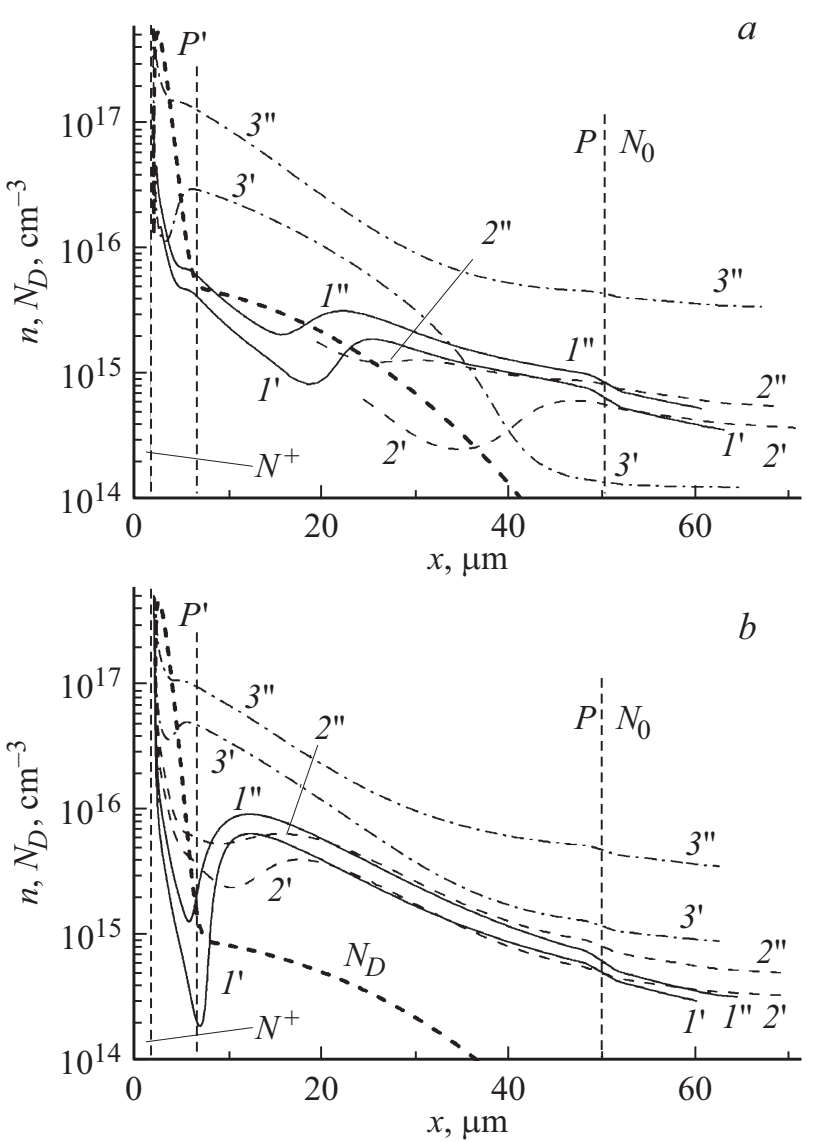

Рис. 3. Распределения концентраций электронов $n$ в катодной окрестности центрального, $S^{\prime}$, и периферийного, $S^{\prime \prime}$, сегментов РВД в различные моменты времени $t_{3}>t_{2}>t_{1}>0$. Жирные штриховые линии - профили легирования $N_{D}$ двуслойной $P$-базы для двух исследуемых структур (см. пояснения в тексте).

начиная с 1.5 раз на стадии накачки, быстро усиливается на стадии обострения выброса напряжения на кривой 1 и доходит до 20 раз к моменту максимума рабочего импульса $I(t)$. Практически весь ток за это время 
вытесняется из центральной части $\mathbf{S}^{\prime}$ в периферийный сегмент $\mathbf{S}^{\prime \prime}$, так что его плотность здесь практически удваивается. Переходный пик напряжения при $t \approx 30 \mathrm{Hс}$ достигает максимума $U_{m}=1.2 \mathrm{\kappa B}$, что уже соизмеримо с рабочим напряжением для прототипа из [10] и говорит о перегрузке по току. Именно в интервале времен 20-40 нс, где одновременно велики $U(t)$ и $I(t)$, происходит быстрый прирост интегралов потерь, разный по темпу в разных сегментах. В результате к концу импульса интегральные потери в сегменте $\mathbf{S}^{\prime}$ оказываются почти в 8 раз меньшими, чем в $\mathbf{S}^{\prime \prime}$.

\section{2. „Вертикальная“ динамика инжекции в коаксиальных центральном и периферийном сегментах}

Отмеченные особенности временни́х разверток $U(t)$, $I(t)$ и $W(t)$ могут быть объяснены на основе анализа $x$-профилей распределений инжектированных электронов в двух выделенных сегментах чипа. На рис. 3, $a$ приведены результаты имитационного моделирования вертикальной динамики инжекции для случая с неоптимизированным легированием многослойной структуры и недостаточно интенсивной накачкой $\left(N_{s}=5 \cdot 10^{15} \mathrm{~cm}^{-3}\right.$, $Q_{R}=3.75$ мкКл) при температуре сегментов $T^{\prime}=420$ и $T^{\prime \prime}=380 \mathrm{~K}$. Распределения электронных концентраций показаны для моментов $t_{1}=16, t_{2}=20$ и $t_{3}=110 \mathrm{Hc}$ кривыми $1^{\prime}-3^{\prime}$ и $1^{\prime \prime}-3^{\prime \prime}$, соответствующими начальной стадии формирования фронта включения, переходу к резкому обострению импульса $U(t)$ и прохождению максимума импульса тока $I(t)$ на рис. 2. Видно, как к моменту $t_{1}$ в $P$-базе катодного транзистора уже появляются участки концентрационного обеднения, расширяющиеся к моменту $t_{2}$ к аноду за счет оттеснения накопленной там плазмы при едва начинающемся инжекционном пополнении электронов из катода (происходит трансформация форм $I^{\prime} \rightarrow 2^{\prime}$ и $\left.I^{\prime \prime} \rightarrow 2^{\prime \prime}\right)$. При этом из-за более сильного температурного подавления инжекции в сегменте $\mathbf{S}^{\prime}$ его $P$-база истощается раньше, а начинает пополняться позже, чем в сегменте $\mathbf{S}^{\prime \prime}$.

Для $t>t_{2}$ характер трансформаций электронных профилей в $\mathbf{S}^{\prime}$ и $\mathbf{S}^{\prime \prime}$ резко меняется. Если в периферийной части происходит усиление инжекционной модуляции по всему объему сегмента (переход $2^{\prime \prime} \rightarrow 3^{\prime \prime}$ ), то в сегменте $\mathbf{S}^{\prime}$ область пониженной концентрации проникает в $N_{0}$-базу при ограниченном пополнении электронами катодной окрестности $P$-базы (переход $2^{\prime} \rightarrow 3^{\prime}$ ). Заметим, что к моменту $t_{3}$ минимальные концентрации электронов в сегменте $\mathbf{S}^{\prime \prime}$ не поднимаются выше $(2-5) \cdot 10^{15} \mathrm{~cm}^{-3}$, что на порядок ниже, чем в микросекундных РВД. Это свидетельствует о незавершенности процесса концентрационной модуляции прибора в описанном случае.

Заметно по-другому протекает начальная стадия перераспределяемой по сегментам инжекции в модифицированном РВД-чипе со сниженным легированием $N_{s}=1 \cdot 10^{15} \mathrm{~cm}^{-3}$ и повышенном уровне накачки
$Q_{R}=5$ мкКл (рис. $3, b$ ) при температуре сегментов $T^{\prime}=420$ и $T^{\prime \prime}=370 \mathrm{~K}$. Некоторое оттеснение электронов, введенных при накачке, и задержка поступления новых электронов из катода к моменту $t_{1}=10$ мкс (кривые $1^{\prime}, 1^{\prime \prime}$ ) наблюдаются только в узком $P^{\prime}$-слое базы катодного транзистора. Провалы концентраций на $l^{\prime}, l^{\prime \prime}$ сглаживаются к моменту $t_{2}=20$ мкс, и для $t>t_{2}$ уровень инжекции в обоих сегментах становится высоким всюду в $P$ - и $N_{0}$-слоях по обе стороны перехода коллектора. При этом в период между $t_{2}$ и $t_{3}$ резкого „переброса“ формы профиля электронов с демодуляцией концентраций в сегменте $\mathbf{S}^{\prime}$ вообще не возникает. Эту ситуацию следует классифицировать как субмикросекундную реализацию квазидиодного режима работы динисторов - понятия, введенного в обиход в работе [2] для характеризации оптимального запуска микросекундных РВД.

Можно ожидать, что эффекты динамического перераспределения токов и тепловых потерь в РВД с поперечным перепадом температуры для квазидиодных режимов работы будут выражены гораздо слабее (см. расчеты далее в разд. 4.1).

\section{3. Радиальное распределение температуры В слое Т при заданной листовой мощности тепловых потерь в слое $\mathrm{S}$}

В принципе, при известных радиальных распределениях температуры и листовой плотности мощности тепловых потерь $W(r)$ в слое $\mathbf{S}$ и заданном apriori пробном значении частоты $f^{*}$ можно определить среднюю за время $\tau_{f}$ объемную плотность мощности тепла, генерируемого по радиусу пластины теплоотвода $\mathbf{T}$ :

$$
\langle P\rangle=f^{*} W / h .
$$

Стационарный баланс тепла в слое $\mathbf{T}$, пополняемого этим „вертикальным“ его притоком $\langle P\rangle$ и отводимого на периферийную границу $r=r_{m}$ с внешним радиатором листовым радиальным потоком $\Phi$, описывается стационарным уравнением теплопроводности в слое $\mathbf{T}$

$$
\frac{1}{r} \frac{d}{d r}\left(r \frac{d}{d r} T^{*}\right)=-\frac{\langle P\rangle}{\kappa},
$$

где $\kappa$ - теплопроводность материала теплоотвода.

Общая форма искомого радиального распределения температуры $T^{*}\left(r, W, f^{*}\right)$ может быть найдена в виде квадратуры для любых радиальных профилей $W(r)$ :

$$
T^{*}\left(r, W, f^{*}\right)=\int_{r} \frac{r}{2 \kappa} \int_{r}\langle P\rangle d \rho d r .
$$

Конкретное решение для $T^{*}$ отбирается в соответствии с граничными условиями $d T /\left.d r\right|_{r=0}=0$ и $\left.T\right|_{r=r_{m}}=T_{\min }$, из которых первое отражает цилиндрическую симметрию задачи, а второе определено температурой на границе с внешним радиатором и может варьироваться в рамках доступных технических пределов. Так, 


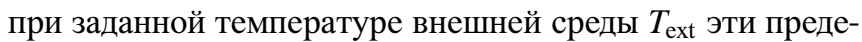
лы определяются тепловым сопротивлением радиатора $R_{T}^{\text {ext }}$, приведенным к единичному охлаждаемому модулю, и граничное условие приобретает вид

$$
\left.\kappa(d T / d r)\right|_{r=r_{m}}=-\left(\left.T\right|_{r=r_{m}}-T_{\mathrm{ext}}\right) / R_{T}^{\mathrm{ext}} .
$$

При двухступенчатой аппроксимации температур дискретных сегментов $T^{\prime}$ и $T^{\prime \prime}$ явные выражения для $T^{*}$ имеют вид $T_{1}(r)=T_{\max }-a\left\langle P_{1}\right\rangle r^{2} / 2 \kappa$ для $0<r<r^{\prime}$ и $T_{2}(r)=T_{1}\left(r^{\prime}\right)-c\left\langle P_{1}\right\rangle r / \kappa-d\left\langle P_{2}\right\rangle r^{2} / 2 \kappa$ для $r^{\prime}<r<r_{m}$. Полная форма этих выражений с раскрытием зависимостей коэффициентов $a, c, d$ от $f^{*}$ и $T_{\min }$ достаточно громоздка и здесь не раскрывается.

В нашем случае усредненные по площадям отдельных сегментов $\mathbf{S}^{\prime}$ и $\mathbf{S}^{\prime \prime}$ и зависящие от параметров $f^{*}$ и $T_{\min }$ значения $T_{1}$ и $T_{2}$, которые понадобятся далее для выполнения итерационного согласования инжекционной и тепловой задач, находятся при интегрировании распределений (6) или их аналитических эквивалентов по формуле

$$
T_{1,2}=2 \pi\left(A^{\prime}, A^{\prime \prime}\right)^{-1} \int_{A^{\prime}, A^{\prime \prime}} T(r) r d r .
$$

\section{4. Результаты численного анализа}

\section{1. Эффекты интенсивности реверсивной накачки}

Обсудим вопрос о влиянии поперечного перепада температуры на некоторые показатели эффективности импульса реверсивной накачки - величины переходных пиков $U_{m}$ и интегралов тепловых потерь $W$ (рис. 4).

Сначала рассмотрим характеристики конструкции, в которой $P^{\prime}$-слой базы катодного транзистора легирован акцепторами с поверхностной концентрацией $N_{s}=5 \cdot 10^{15} \mathrm{~cm}^{-3}$, при температурах сегментов $T^{\prime}=420$ и $T^{\prime \prime}=380 \mathrm{~K}$. В этом случае при величине заряда реверсивной накачки $Q_{R} \approx 3$ мкКл наблюдается переходный пик напряжения $U_{m} \approx 2$ кВ (кривая 1 ), близкий по высоте к рабочему напряжению прототипа РВД-1 (см. разд. 3.1). Хотя высота пика снижается до $700 \mathrm{~B}$ при $Q_{R} \approx 4.8$ мкКл (уровень накачки прототипа в [10]), суммарные интегральные потери в центральном, $W^{\prime}$, и в периферийном, $W^{\prime \prime}$, сегментах (соответственно кривые $3^{\prime}$ и $3^{\prime \prime}$ ) при этом оказываются в полтора раза бо́льшими, чем в однородно нагретом прототипе при $T=400 \mathrm{~K}$. Это преобладание по тепловым потерям остается на четверть бо́льшим при увеличении заряда накачки до 10 мкКл, что свидетельствует о недостаточной оптимизации исследуемого образца (см. в рабо$\operatorname{Tax}[10,11])$.

Ситуация заметно улучшается при снижении параметра поверхностной концентрации легирования профиля до $N_{s}=1 \cdot 10^{15} \mathrm{~cm}^{-3}$ даже при несколько расширенном перепаде температуры $\delta T \rightarrow 50 \mathrm{~K}$. Генерируемый пик

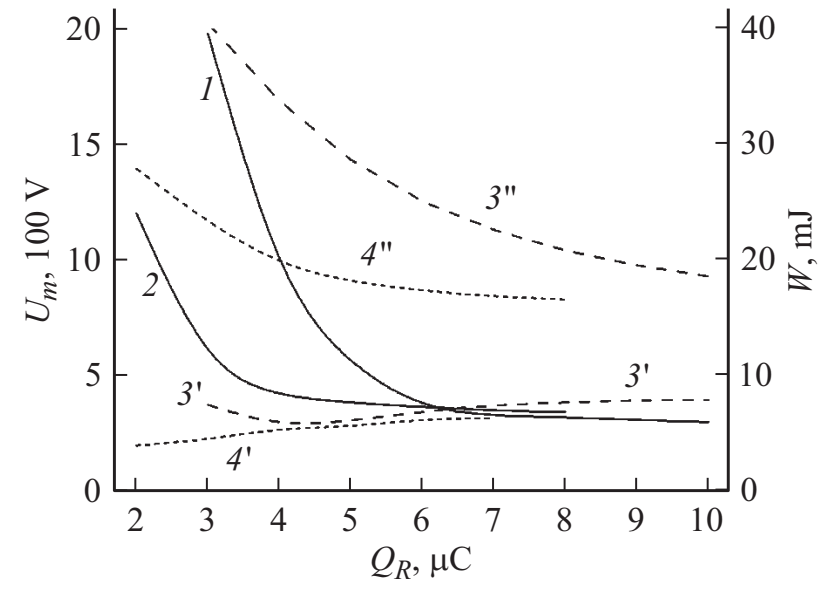

Рис. 4. Зависимости высоты пика напряжения $U_{m}$ (сплошные кривые) и интегралов тепловых потерь $W$ (штриховые) от заряда реверсивной накачки $Q_{R}$ при различных значениях параметра $N_{s}$ и температур сегментов $T^{\prime}$ и $T^{\prime \prime}$ (см. обозначения в тексте).

напряжения $U_{m} \approx 1.2$ кВ (кривая 1 ) теперь не превосходит и половины рабочего напряжения прототипа даже при $Q_{R} \rightarrow 2$ мкКл. Он вдвое снижается уже при $Q_{R} \approx 3$ мкКл, а при $Q_{R}>4-5$ мкКл насыщается к минимальному уровню $\sim 350 \mathrm{~B}$, что по определению теории [2] является наблюдаемым признаком реализации „квазидиодного“ скоростного предела для РВД. Насыщенные значения потерь $W^{\prime}$ и $W^{\prime \prime}$ (кривые $4^{\prime}$ и $4^{\prime \prime}$ ) в сумме теперь всего на $10-15 \%$ выше, чем в однородно нагретом прототипе.

\section{2. Эффекты радиального перепада температуры}

Для количественного описания зависимостей электротепловых эффектов от перепада температуры $\delta T=T^{\prime}-T^{\prime \prime}$ в оптимизированных модификациях РВД было выполнено их имитационное компьютерное моделирование для 15 точек из интервала изменения $\{\delta T\}=\{0,60 \mathrm{~K}\}$. Результаты расчета для конструкции чипа РВД с поверхностной концентрацией профиля легирования $P$-базы $N_{p s}=1 \cdot 10^{15} \mathrm{~cm}^{-3}$ представлены на рис. 5. Величина заряда накачки к моменту $t=0$ составляла $Q_{R}=5$ мкКл. Температура сегмента $T^{\prime}=420 \mathrm{~K}$ полагалась фиксированной и примерно равной максимально допустимой рабочей температуре. Форма импульса $I(t)$ в период $t>0$ задавалась по выражению (2).

Заметим, что наблюдаемое на рис. 5 снижение величин переходных пиков $U_{m}$ и джоулевых потерь за импульс $W$ с увеличением $\delta T$ было вполне ожидаемым. При этом количественная адекватность расчета зависимостей $W(\delta T)$ гарантируется высоким уровнем апробации известного программного продукта [15]. Последующее использование полученных здесь количественных данных о тепловых потерях в чипе РВД в аналитическом описании работы теплоотвода по выражениям (5)-(7) 


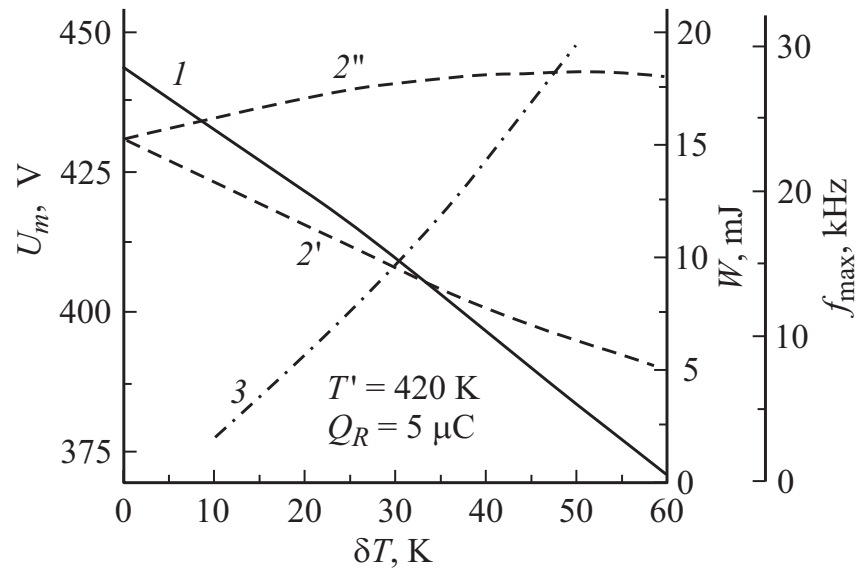

Рис. 5. Зависимости от перепада температуры $\delta T=T^{\prime}-T^{\prime \prime}$ величин пикового напряжения $U_{m}$ на чипе $(1)$ и джоулевых потерь энергии за импульс $W^{\prime}\left(2^{\prime}\right)$ и $W^{\prime \prime}\left(2^{\prime \prime}\right)$ в сегментах $\mathbf{S}^{\prime}$ и $\mathbf{S}^{\prime \prime}$, а также максимально допустимой частоты генератора $f_{\max }(3)$ при параметрах теплоотвода $h=0.1 \mathrm{~cm}$ и $\kappa=3.9 \mathrm{BT} \cdot \mathrm{cm}^{-1} \cdot \mathrm{K}^{-1}$ (медь).

дает возможность выполнить надежный расчет максимальной допустимой частоты повторения импульсов по объединенной методике, изложенной далее.

\section{3. Расчет рабочей частоты}

В произвольном случае величины $T_{1}$ и $T_{2}$, вычисляемые по теории в разд. 3.3, не совпадают с заданными apriori значениями $T^{\prime}$ и $T^{\prime \prime}$. Чтобы окончательно установить взаимно-однозначное соответствие между внешними показателями РВД-модуля $f_{\max }$ и $T_{\min }$, мы используем сходящуюся итерационную процедуру, в которой пробные значения $T_{\min }$ (значит и $T_{1}, T_{2}$ ) и первично назначаемые параметры $f^{*}, T^{\prime}, T^{\prime \prime}$ пошагово корректируются до достижения приемлемого уровня минимизации разностей $T_{1}-T^{\prime}$ и $T_{2}-T^{\prime \prime}$.

Рассчитанная этим способом зависимость максимальной допустимой частоты высоковольтного генератора (кривая 3 на рис. 5) от перепада температуры $\delta T$ указывает на возможность ее изменения на порядок в диапазоне 3-30 кГц при таком усилении интенсивности внешнего охлаждения, чтобы было обеспечено увеличение поперечного перепада температуры от 10 до $50 \mathrm{~K}$. Необходимая для этого мощность теплового потока во внешний радиатор, отнесенная к единичному звену, оценивается как $\Phi_{\text {out }}=\Phi^{\prime \prime}=f_{\max }\left(W^{\prime}+W^{\prime \prime}\right)$ и лежит в диапазоне 90-750 Вт.

\section{5. Заключение}

В работе развит новый расчетно-теоретический подход к исследованию коммутационных и тепловых физических процессов в реверсивно-включаемых динисторах, функционирующих в установившихся режимах частотно- го повторения импульсов при ограниченной интенсивности систем импульсного управления и отвода джоулева тепла. Обоснован способ радикального упрощения многомасштабной задачи описания самосогласованного нелинейного взаимодействия инжекционной и теплоотводящей подсистем, и на его основе предложен метод расчета максимальной частоты РВД-коммутатора.

На примере модифицированной конструкции РВД с шестислойной $N^{+} P^{\prime} P N_{0} N^{\prime} P^{+}$-структурой, рассчитанной на рабочее напряжение $2.5 \mathrm{~KB}$, показано, что подобные модификации с чипом площадью $1 \mathrm{~cm}^{2}$ способны при умеренной величине заряда реверсивной накачки 4-5 мкКл коммутировать импульсы тока длительностью в сотни наносекунд, передающие в нагрузку энергию 0.25 Дж за импульс с частотой повторения до 30 кГц. Для собранных на таких чипах частотных РВД-генераторов с напряжением 100 кВ и выше это соответствует передаваемой мощности в доли МВт.

Описанная методика расчета быстродействующих конструкций РВД в условиях с поперечным перепадом температуры вместе с оценками допустимых частот высоковольтных генераторов на их основе может послужить эскизной схемой для построения полной базы данных, необходимых при разработке новых модификаций РВД.

Работа выполнена при поддержке РНФ, проект № 14-29-00094.

\section{Список литературы}

[1] I.V. Grekhov, A.V. Gorbatyuk, L.S. Kostina, S.V. Korotkov, N.S. Jakovtchuk. Solid-State Electron., 26 (11), 1132 (1983).

[2] A.V. Gorbatyuk, I.V. Grekhov, A.V. Nalivkin. Solid-State Electron., 31 (10), 1483 (1988).

[3] С.В. Коротков. ПТЭ, № 4, 5 (2002).

[4] T. Bertier, V.G. Bezuglov, I.V. Galakhov, S.N. Gudov, J.-P. Marret, V.M. Murugov, V.A. Ostin, D. Rubin de Cervens, M.L. Smetanin, V.I. Zolotovski. Proc. XIIth IEEE Int. Pulse Power Conf. (Monterey, 1999) p. 914.

[5] M.E. Savage. IEEE Trans. Plasma Sci., 28 (5), 1451 (2000).

[6] S. Schneider, T.F. Podlesak. IEEE Trans. Plasma Sci., 28 (5), 1520 (2000).

[7] H. Wang, X. He, W. Chen, L. Xie, J. Zhou, B. Xue, F. Guo, G. Zhang, Z. Chen, Z. Zeng. IEEE Trans. Pow. Electron., 29 (4), 1553 ( 2014).

[8] С.В. Коротков, А.Л. Жмодиков. ПТЭ, № 1, 68 (2011).

[9] С.В. Коротков, Ю.В. Аристов, Д.А. Коротков. ПТЭ, № 3, 94 (2012).

[10] А.В. Горбатюк, Б.В. Иванов. ЖТФ, 85 (8), 94 (2015).

[11] А.В. Горбатюк, Б.В. Иванов. ФТП, 51 (6), 835 (2017).

[12] А.В. Горбатюк, Б.В. Иванов. Письма ПЖТФ, $45(1), 57$ (2019).

[13] И.В. Грехов, Г.А. Месяц. УФН, 175 (7), 736 (2005).

[14] А.А. Самарский, А.П. Михайлов. Математическое моделирование (М., Физматлит, 2002).

[15] Synopsys Dev. Simulation, TCAD Sentaurus, manual. http://www.synopsys.com

Редактор Л.В. Шаронова 


\title{
Inhomogeneous injection and heat transfer processes in reversely switched dynistors working in pulse frequency repetition modes with limited heat sink
}

\author{
A.V. Gorbatyuk ${ }^{1}$, B.V. Ivanov ${ }^{2}$ \\ ${ }^{1}$ loffe Institute, \\ 194021 St. Petersburg, Russia \\ 2 St. Petersburg State Electrotechnical University, \\ 197376 St. Petersburg, Russia
}

\begin{abstract}
For the first time, a self-consistent computationaltheoretical description of physical processes in reversely-switched dynistors (RSD) is obtained when operating in frequency pulserepetition modes with a limited heat sink. A simplified representation of the nonlinear multiscale mechanism of interaction between the injection and heat transfer subsystems is substantiated, and on this basis a method for calculating the maximal frequency of the RSD switching unit is developed. The dependence of the permissible frequency on the power of the cooler for the given parameters of the chip and the shape of the switched pulses is calculated. It is shown that with a proper heat removal, unit RSD-modules with a chip area of $1 \mathrm{~cm}^{2}$ and an operating voltage of $U \approx 2.5 \mathrm{kV}$ will be able to switch the energy of $0.25 \mathrm{~J}$ per pulse with a repetition frequency of up to $30 \mathrm{kHz}$. For high-voltage generators based on them with $U \approx 100 \mathrm{kV}$ power transmitted to the load at this frequency will be several fractions of MW.
\end{abstract}

